\title{
Actividade física e práticas de lazer em adolescentes
}

\author{
Carlos Esculcas \\ Jorge Mota
}

https://doi.org/10.5628/rpcd.05.01.69

\author{
Universidade do Porto \\ Faculdade de Ciências do Desporto e de Educação Física \\ Centro de Investigação em Actividade Física, Saúde e Lazer
}

\section{RESUMO}

Os objectivos do presente trabalho foram: (i) Identificar quais as actividades de lazer mais procuradas pelos adolescentes; (ii) Apreciar os mecanismos de influências da idade e do género na adesão às práticas de lazer, como opção livre e espontânea, durante a adolescência. Foram avaliados 594 sujeitos (15,9 \pm 2,18 anos), sendo 304 do sexo feminino e 290 do sexo masculino. As actividades de lazer foram avaliadas por intermédio de um inventário que pretendeu identificar as actividades habituais de lazer dos jovens adolescentes. Os nossos resultados indicam que os adolescentes têm uma grande componente de tempo livre não estruturado. As actividades praticadas pela proporção mais elevada dos jovens podem ser classificadas como lazeres não activos, verificando-se que a participação em actividades físicas não organizadas é apenas indicada em décimo quarto lugar, com $42.2 \%$, e as não organizadas em décimo quinto lugar, com $41 \%$ das respostas. Em relação ao género, catorze das vinte e uma actividades inventariadas apresentam diferenças significativas com excepção de «Ouvir música», «Ver televisão ou vídeo", "Visitar pessoas conhecidas", «Ir ao cinema, concertos, teatro", "Trabalhar para ganhar algum dinheiro", "Actividades de arte e expressão" e "Trabalhos de solidariedade social». As diferenças de género e etárias devem ser tidas em conta ao nível dos programas de intervenção, no sentido de favorecer práticas tidas como mais positivas no tempo de lazer da população infanto-juvenil.

Palavras-chave: actividade física, lazer, adolescência

\section{ABSTRACT \\ Physical and Leisure Activities in Adolescents}

The main purposes of this study were (i) identify the most frequent leisure activities; (ii) examine the influence of age and gender on those. The sample comprised 594 adolescents $(15.9 \pm 2,18$ years-old $)$, 304 females and 290 males. Leisure activities were assessed by questionnaire. The findings showed that adolescents had strong non-structured leisure-time activities. Most activities were sedentary ones.

Gender-related differences were found in 14 over 21 reported activities. Gender and age differences should be taken into account when defined intervention programs

Key Words: physical activity, leisure, adolescence. 


\section{INTRODUÇÃO}

A actividade física, como uma actividade realizada durante o tempo discricionário (perspectiva objectiva do lazer), pode levar a um aumento significativo no dispêndio energético com potenciais influências no estado de saúde das pessoas.

Nas práticas de lazer, o ponto central é o sentimento positivo que o sujeito possui para com a actividade (25), tendo a investigação quantitativa demonstrado claramente que o comportamento dos jovens não é apenas influenciado pelas experiências objectivas, mas também pela percepção dessas experiências (17). Por isso, se a experiência for positiva ou divertida, se a experiência valorizar as percepções das crianças e dos jovens, então, com mais probabilidade se manterá a actividade para o resto da sua vida (15). A acessibilidade às práticas de actividade física no tempo de lazer é claramente dependente de um conjunto de factores, entre os quais o sexo e a idade. Assim, os objectivos do presente trabalho foram: (i) Identificar quais as actividades de lazer mais procuradas pelos adolescentes; (ii) Apreciar os mecanismos de influências da idade e do género na adesão às práticas de lazer, como opção livre e espontânea, durante a adolescência.

\section{MATERIAL E MÉTODOS \\ Amostra}

A amostra foi constituída por 594 alunos que frequentavam os níveis de escolaridade compreendidos entre o $6^{\circ}$ e o $12^{\circ}$ anos de escolaridade.

As idades dos sujeitos estão compreendidas entre os doze e os vinte anos $(15,9 \pm 2,18)$, sendo 304 do sexo feminino e 290 do sexo masculino, constituindo respectivamente $51,2 \%$ e $48,8 \%$ do total da amostra.

Três sub-amostras foram constituídas para os grupos de idade (Tabela 1), procurando-se que cada um dos intervalos etários estivesse representado no total da amostra com o mesmo número de sujeitos. Também para o sexo se seguiu o mesmo procedimento, tanto em relação à representatividade de cada um dos grupos, como entre grupos.

Foram distribuídos 2.000 questionários, verificandose o retorno de 1.013. O não retorno deveu-se ao extravio dos instrumentos de aferição e à entrega dos mesmos em tempo não-útil para que se pudesse realizar a constituição da base de dados e análises estatísticas. Dos 1.013 questionários referidos foram expurgados 419 sujeitos, para que fosse possível manter os critérios de representatividade para os intervalos de idade e sexo, bem como pela exclusão de questionários incompletos.

Tabela 1 - Distribuição do sexo pelos grupos de idade.

\begin{tabular}{lccc}
\cline { 2 - 4 } & \multicolumn{3}{c}{ Intervalos de idade } \\
& $\begin{array}{c}12-14 \\
{[\mathrm{n}=198)}\end{array}$ & $\begin{array}{c}15-17 \\
{[\mathrm{n}=198)}\end{array}$ & $\begin{array}{c}18-20 \\
{[\mathrm{n}=198]}\end{array}$ \\
Sexo & & & \\
Masculino & $33,6 \%$ & $32,6 \%$ & $33,9 \%$ \\
Feminino & $33,2 \%$ & $34,3 \%$ & $32,5 \%$ \\
Total & $33,4 \%$ & $33,4 \%$ & $33,4 \%$
\end{tabular}

\section{Instrumentos}

As actividades de lazer foram avaliadas por intermédio de um inventário que pretendeu identificar as actividades habituais de lazer dos jovens adolescentes (4). Com este objectivo apresentou-se uma lista de 21 actividades, das quais os sujeitos seleccionaram aquelas a que aderiram ou não. Para a adaptação do inventário foram realizadas as seguintes diligências: (i) Tradução para a língua portuguesa efectuada por três professoras de Português/Francês do ensino secundário. Cada uma das docentes efectuou uma tradução individual, realizando-se posteriormente a comparação entre as traduções e a expurgação dos elementos não concordantes; (ii) Aplicação do questionário a 60 sujeitos, com o objectivo de avaliar a exequibilidade da sua aplicação. Procurou-se aferir o tempo de preenchimento, compreensão e subjectividade de interpretação, efectuando-se, posteriormente, a determinação e correcção dos elementos ambíguos; (iii) Análise da variabilidade das respostas.

\section{Procedimentos estatísticos}

As análises estatísticas foram efectuadas com recurso ao programa estatístico S.P.S.S. (Statistical Package for the Social Sciences) para o Windows, versão 10.0. Foi efectuado o teste de tendência de Jonckheere (valor S) para avaliação das tendências das práticas de lazer durante a adolescência considerando para esse efeito os escalóes etários definidos. Foi ainda utilizado o teste do qui-quadrado $\left(\chi^{2}\right)$ para a determinação das diferenças entre as variáveis nominais, nomeadamente o sexo. O nível de significância foi colocado em $5 \%$. 


\section{RESULTADOS}

As práticas de lazer dos adolescentes, bem como as tendências observadas ao longo da adolescência, encontram-se resumidas na tabela 2. Note-se que o teste estatístico utilizado (valor para o teste de Jonckheere-Terpstra) apresenta valores positivos e negativos, significando, neste caso concreto, os valores positivos uma diminuição da participação na actividade considerada e os valores negativos o aumento da participação nessa actividade, desde o intervalo de idade mais jovem (12-14) até ao mais velho (18-20). As práticas que apresentam uma tendência positiva (no sentido atrás referido) estatisticamente significativa para os diferentes intervalos de idade são: "A realização de trabalhos para a escola» $(S=2.270)$, observando-se uma diminuição da participação nesta actividade ao longo da adolescência; «Ler» $(\mathrm{S}=$ 3.542), com o abandono pronunciado $(\mathrm{p}<0.001)$ dos hábitos de leitura; "Jogos de Vídeo, cartas e computador", também com uma significância de 0.001; «Visitar pessoas conhecidas» $(\mathrm{S}=4.418, \mathrm{p}<0.05)$. Com tendência negativa significativa (neste caso, aumento do envolvimento na actividade) observam- se as seguintes actividades: "Namorar; estar com o namorado $(\mathrm{a})$ " $(\mathrm{S}=-3.989)$ e "Ir à discoteca» $(\mathrm{S}=$ 4.727), ambas com um $\mathrm{p}<0.001$.

No conjunto dos sujeitos, a proporção dos jovens que declara praticar regularmente as diferentes actividades de lazer varia entre 94.3\% (Ouvir música) e $16.9 \%$ (Trabalho de solidariedade social). Quatro tipos de actividades são apontadas, em média, por mais de nove em dez adolescentes: "Ouvir música», «Trabalhos para a escola», "Ver televisão ou vídeo» e "Conversar com os amigos».

É importante realçar o facto de que, das quatro práticas (em média) de lazer mais apontadas (entre $94.3 \%$ e $90.1 \%$ ), apenas os «trabalhos realizados para a escola» apresentam uma tendência significativa de abandono, mantendo-se os elevados índices percentuais para as outras actividades, em todos os escalões etários. Assim, as actividades praticadas pela proporção mais elevada dos jovens podem ser classificadas como lazeres não activos, verificando-se que a participação em actividades físicas não organizadas é apenas indicada em décimo quarto lugar, com $42.2 \%$, e as não organizadas em décimo quinto lugar, com $41 \%$ das respostas.

Tabela 2 - Tendências (Teste Jonckheere-Terpstra) das práticas de lazer para os intervalos de idade e médias das práticas para a população.

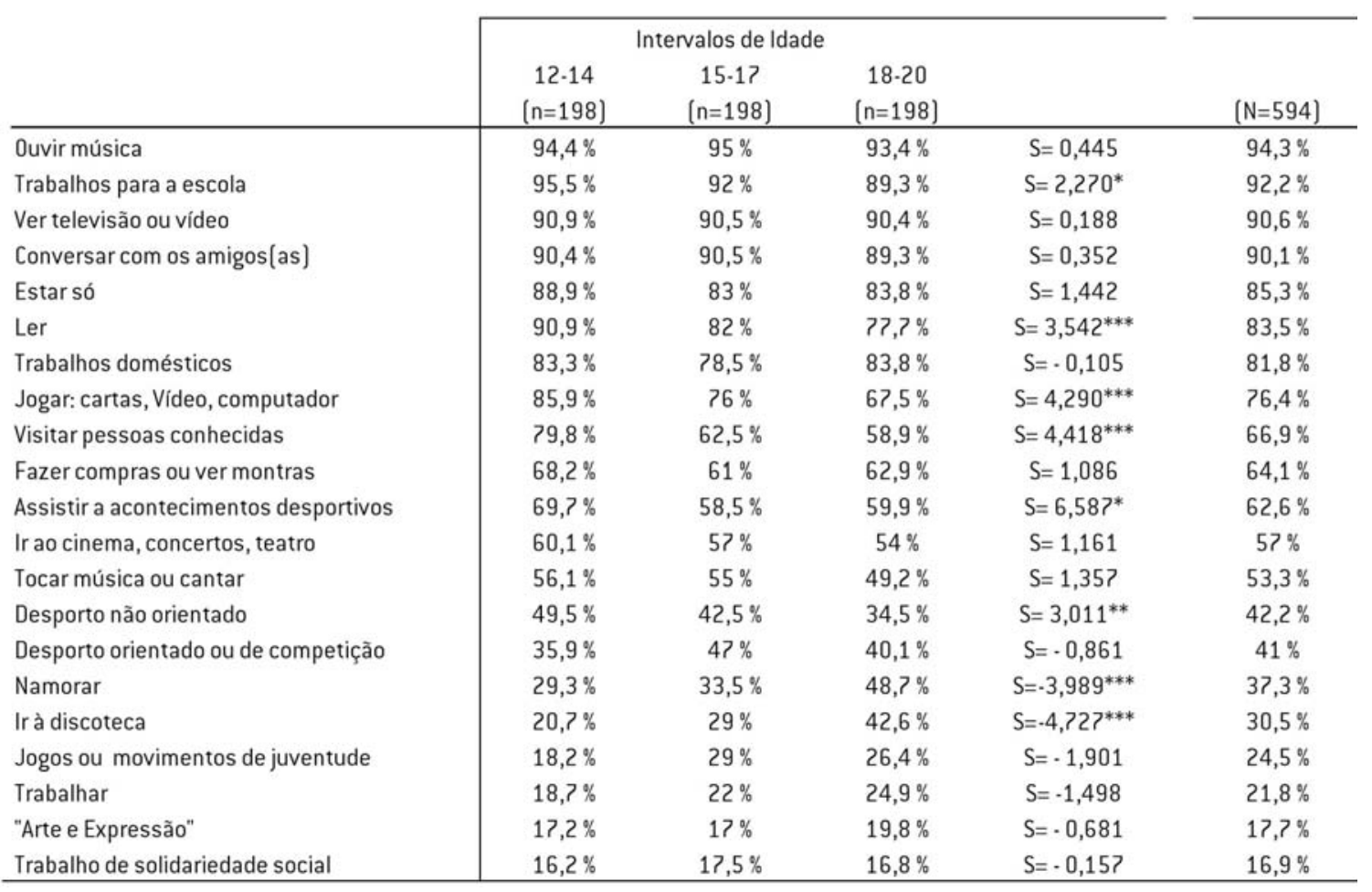


Duas das actividades que de alguma forma poderão estar associadas a estilos de vida activos ("Assistir a acontecimentos desportivos» e "Desporto não orientado») apresentam um teste de tendência significativamente positivo (no caso, abandono da actividade considerada), com particular realce para o segundo: de uma participação de $49.5 \%$ no intervalo de idade dos 12-13 anos, a percentagem para «desporto não orientado" situa-se nos $34.5 \%$ para os $18-20$ anos $(\mathrm{p}<0.01)$. Em relação às "práticas orientadas/dirigidas ou de competição", apesar do teste não ser significativo, a tendência para o abandono destas actividades não se verifica com o decorrer da idade. Os resultados parecem indicar que a participação em actividades orientadas/dirigidas aumenta no grupo de idade intermédio (15-17 anos), seguindo-se o abandono das mesmas no grupo dos 18-20 anos.
A situação contrária verifica-se nas actividades não-orientadas, isto é, o abandono destas actividades é uma tendência com significado estatístico. Note-se, no entanto, que no escalão etário mais jovem (12-14 anos), o valor percentual para as actividades não orientadas (49.5\%) é superior ao das actividades orientadas/dirigidas (35.9\%), sendo esta diferença entre participantes, num e noutro tipo de actividade física, suficiente para "induzir" uma média global superior para as actividades não-orientadas ( $42.2 \%$ e $41 \%$ para as orientadas/dirigidas). Em relação ao género (Tabela 3 ), catorze das vinte e uma actividades inventariadas apresentam diferenças significativas com excepção de "Ouvir música», "Ver televisão ou vídeo», «Visitar pessoas conhecidas», "Ir ao cinema, concertos, teatro», "Trabalhar para ganhar algum dinheiro", "Actividades de Arte e Expressão»e «Trabalhos de solidariedade social».

Tabela 3 - Diferenças das práticas de lazer para o género.

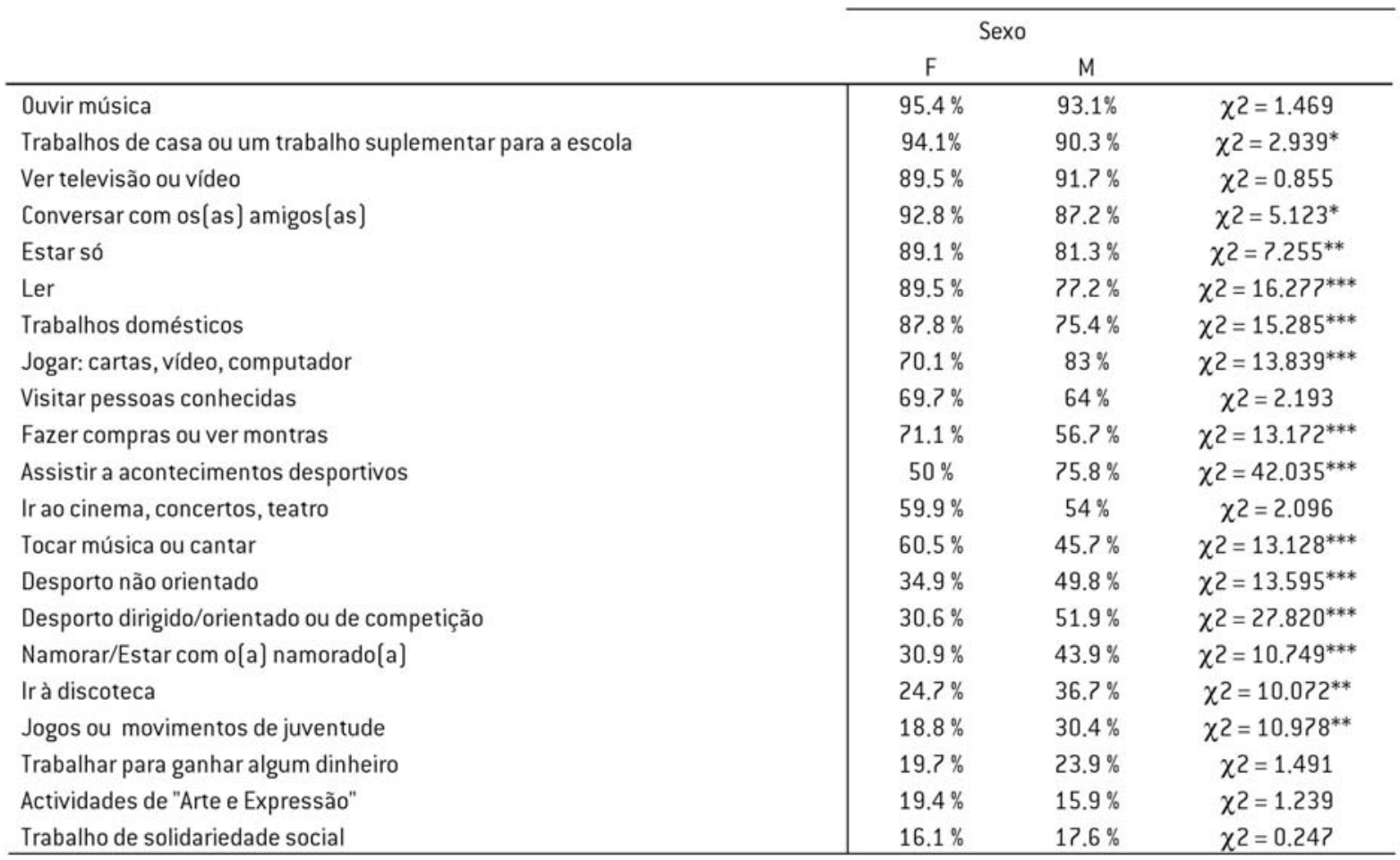


Apesar da ausência da significância estatística para estas actividades, verifica-se que as percentagens observadas no sexo feminino são superiores nas actividades "Ouvir música» (95.4\% para $93.1 \%$ dos rapazes), «Visitar pessoas conhecidas» (69.7\% para $64 \%$ dos rapazes), «Ir ao cinema, concertos, teatro» ( $59.9 \%$ para $54 \%$ dos rapazes) e "Actividades de arte e expressão" (19.4 \% para $15.9 \%$ dos rapazes), o que denota preponderâncias nas práticas de lazer diversas dos rapazes. Estes últimos apresentam percentagens superiores nas actividades "Ver televisão ou vídeo" (91.7 \% para $89.5 \%$ das raparigas), «Jogar: cartas, computador, vídeo-games», «Trabalhar para ganhar algum dinheiro" (23.9\% para $19.7 \%$ para as raparigas) e "Trabalho de solidariedade social» ( $17.6 \%$ para $16.1 \%$ para as raparigas).

\section{DISCUSSÃO}

Considerando a repartição das categorias de lazer em função das percentagens médias de jovens praticantes, a análise comparativa entre o presente estudo e o efectuado por Cloes et al. (4) para os jovens europeus (recorrendo ambos exactamente ao mesmo inventário de actividades), permitiu verificar que, nas sete primeiras actividades indicadas, apenas duas delas não coincidiram em termos de graduação, a saber: a "realização dos trabalhos para a escola" $\left(2^{\mathrm{a}}\right.$ escolha, com 92,2\% de aderentes, no presente trabalho versus $9^{\text {a }}$ escolha, com $50,6 \%$ de aderentes, no estudo de Cloes et al. (4) e a "prática de um desporto dirigido ou de competição" (14 escolha, com $41 \%$ de aderentes, no presente trabalho versus $7^{\text {a }}$ escolha, com $51,9 \%$ de aderentes, no estudo citado). Para além do estudo já referido de Cloes et al. (4), os nossos resultados para as actividades mais referidas pelos adolescentes parecem reproduzir no contexto cultural português os dados divulgados pelo Sports Council (24) em que $98 \%$ da população via TV, $94 \%$ visitava ou conversava com os amigos ou parentes, $86 \%$ ouvia rádio e $67 \%$ ouvia música.

Por conseguinte, e considerando que as actividades que promovem o envolvimento, o desafio e exigem esforço, contribuem positivamente para o desenvolvimento da identidade (21), parece-nos que a relação percentual inventariada de preferências dos nossos adolescentes nem sempre está em consonância com um contributo desenvolvimental positivo da identidade.
Em relação à televisão ( $90.6 \%$ dos sujeitos, no nosso estudo, escolhem esta actividade), por exemplo, Csikszentmihalyi et al. (6) verificaram que embora os adolescentes passassem grande parte do seu tempo a ver televisão, referiam-se aos desportos e aos jogos como sendo as melhores actividades. Ver televisão era a actividade com menor humor positivo, sem desafio, que requeria o nível mais baixo de capacidades e providenciava menos quantidade de controlo pessoal.

Mas, se no estudo anterior as próprias percepções dos sujeitos são negativas em relação a esta actividade, Delle Fave e Bassi (8), num estudo com adolescentes italianos, verificaram que ver televisão é uma actividade que está comummente associada com a apatia e não com a "experiência óptima", sendo a apatia caracterizada pela percepção de um balanço negativo entre os desafios e as competências pessoais (7). Se a televisão apresenta muitas vezes imagens passíveis de serem aprendidas, essas imagens são na maior parte das vezes limitadas por estereótipos e os adolescentes que vêem televisão não estão envolvidos, nem participam de forma real nos contextos sociais, nem nas interacções que observam (21). No extremo oposto à actividade «Ver televisão ou vídeo» encontram-se, com menos adesão, "Jogos ou movimentos de juventude» - $24.5 \%$, «Trabalhar para ganhar algum dinheiro» - $21.8 \%$, «Arte e expressão» - $17.7 \%$ e "Trabalho de solidariedade social» - $16.9 \%$. Esta constatação é precisamente contrária ao referido na literatura em relação às oportunidades (19) que os adolescentes devem ter de educação e treino, formais e informais, realizadas de forma desafiadora e relevante, incluindo a exploração, a prática e a reflexão, bem como a expressão e a criatividade (nosso estudo 17.7\%), e ainda oportunidades que permitam a aquisição de novos papéis e responsabilidades, como a pertença a grupos, prestação de serviços (nosso estudo 16.9\%), ou mesmo, trabalhos pagos em part-time (nosso estudo $21.8 \%$ ). A participação em organizações de juventude (no nosso estudo com apenas $24.5 \%$ de práticas) é também uma das actividades que tende a ser referida por alguns autores, como tendo também grandes benefícios para o desenvolvimento do adolescente (14). Neste tipo de actividades, o adolescente exerce toda uma série de comportamentos e competências 
que se traduzem num enriquecimento pessoal e, consequentemente, numa maior compreensão de si e do mundo que o rodeia. Estão em causa aspectos como a responsabilidade social e mesmo a intervenção social, criando oportunidades para o adolescente questionar e definir os seus próprios valores sociais, ideológicos e de cidadania. Tais aspectos parecem ser fundamentais na construção da identidade (21).

\section{0 género}

Para Hargreaves (12), a construção da divisão de género é uma componente intrínseca da análise cultural, ou seja, do sistema de valores e significados criados activamente pelos indivíduos e grupos. É nesse contexto cultural que se inserem as relações de lazer de ambos os sexos nas diferentes etapas de vida, bem como a existência de diferenças nos padrões masculino e feminino de lazer na nossa sociedade ocidental. Nas mulheres, essas diferenças advêm da tradicional existência doméstica, do nível de escolaridade mais baixo, da função de mãe, dona de casa e esposa, da condição social secundarizada e da situação relativamente protegida das adolescentes (12).

De facto, os estudos empíricos efectuados acerca das práticas de lazer para ambos os sexos parecem sustentar as asserções teóricas desenvolvidas por estes autores.

Assim, numa revisão da literatura efectuada por Fine et al. (9), os autores verificaram que o género é repetidamente referido como um preditor significativo das diferenças nas actividades de lazer dos adolescentes. Parece, então, que os rapazes participam mais em actividades de risco, tais como actividades exteriores e desportos, enquanto as raparigas revelam uma maior participação em actividades de lazer com ênfase em características culturais, sociais e educacionais $(5,11,18)$.

Considerando-se ainda diferentes contextos de lazer, observa-se que as raparigas indicam mais vezes terem tido alguma experiência romântica e de promoção/integração social que os rapazes (23), bem como serem receptoras de mais recompensas sociais pela sua criatividade que os seus pares masculinos $(5,13,20)$.

Estes resultados também foram verificados no nosso estudo aquando da análise das práticas de lazer em função do género. À semelhança, por exemplo, de
Busser (3) - num estudo cujo objectivo era explorar os padrões dos lazeres nos adolescentes e se esses padrões diferiam para o sexo, raça e nível de escolaridade -, verificámos que os rapazes são significativamente mais interessados em actividades de exterior ("Jogos ou movimentos de juventude» - p< 0.001) e desportos ("Assistir a acontecimentos desportivos» $\mathrm{p}<0.001$, «Desporto dirigido/orientado» - $\mathrm{p}<0.001$, «Desporto não dirigido/orientado» - $\mathrm{p}<0.001$ ), enquanto as raparigas orientam os seus interesses para actividades artísticas, desenvolvimento/crescimento pessoal («Estar só» - $\mathrm{p}<0.05$, «Ler» - $\mathrm{p}<$ 0.001, «Trabalhos domésticos» - $p<0.001$, «Trabalhos para a escola» - $\mathrm{p}<0.05)$ e jogos/desportos não tradicionais (34.9\% para os desportos não orientados e $30.6 \%$ para os desportos dirigidos/orientados).

Mas se a análise efectuada por Fitzgerald et al. (10) dos vários tipos de actividades revela, também, diferenças significativas nos interesses em relação ao entretenimento, desportos, actividades sociais e hobbies (actividades com preponderância nos rapazes), estes autores verificaram ainda que as raparigas demonstravam mais interesse pelas actividades de entretenimento, como ir a discotecas, estar com os amigos, conversar com eles e visitá-los. Por outro lado, a preferência pelos "shopping centers" e "shopping" de rua era superior nas raparigas comparativamente aos rapazes (22), bem como a maior preferência dos rapazes pelo cinema do que as raparigas. A especificidade das actividades de lazer participadas pelos e pelas adolescentes referida por estes últimos autores, encontra consonância com os resultados por nós observados. Verificamos que os sujeitos do sexo feminino participam significativamente mais que os rapazes em "Conversar com os amigos(as)" - p< 0.05 e «Fazer compras ou ver montras» - $p<0.001$. Apesar de não se verificarem diferenças significativas para o sexo, as actividades «Visitar pessoas conhecidas» - $69.7 \%$ e "Ir ao cinema, concertos, teatro» $59.9 \%$, revelam preponderância feminina comparativamente aos rapazes ( $64 \%$ e $54 \%$, respectivamente). Neste sentido, parece ser oportuno realçar que as diferenças de género devem também ser tidas em conta ao nível das estratégias de intervenção com jovens, sendo que estas diferenças se relacionam, particularmente, com a auto-estima e com os constrangimentos intrapessoais (1). 


\section{A idade}

Em relação à variável idade os resultados por nós observados encontram também sustentação na literatura. Silbereisen et al. (22), por exemplo, num estudo com dois grupos etários de adolescentes alemães (um com uma idade média de 11.6 anos e o outro com uma idade média de 14.6 anos) verificaram que os sujeitos mais novos preferiam os lugares que permitem a realização de desportos (40\%) enquanto que, nos mais velhos, apesar dos lugares de desporto serem os preferidos, estes só recolhiam $23 \%$ das respostas.

As tendências das práticas de lazer para as actividades físicas/desportivas («Desporto não dirigido/orientado» - $\mathrm{p}<0.01$ e «Desporto dirigido/orientado ou de competição" - apesar de não significativo, verifica-se uma redução percentual do segundo para o terceiro intervalo de idade de $7 \%$ ), nos intervalos de idade no nosso estudo, parecem apontar também no sentido da diminuição da adesão com a idade, particular e significativamente no «Desporto não dirigido/orientado».

Em relação a outras práticas de lazer, os resultados por nós observados parecem coincidir, em grande parte, com o estudo de Silbereisen et al. (23) sobre o desenvolvimento das amizades românticas em adolescentes, naquele caso alemães, e a consequente mudança em relação aos seus contextos de lazer favoritos. Ambos os resultados mostram que os adolescentes mais novos revelam maior preferência pelo contexto do lar (no nosso estudo a adesão às actividades "Ler», "Jogar cartas, vídeo, computador» e «Trabalhos para a escola» que são desenvolvidas comummente em casa, diminuem significativamente - $\mathrm{p}<0.001$ ) havendo uma mudança de interesses para os lugares públicos ao longo do tempo (no nosso estudo a adesão à actividade "Ir à discoteca» aumenta significativamente $-\mathrm{p}<0.001$ ), enquanto que os adolescentes mais velhos mostram uma preferência inicial pelos lugares públicos, para depois manifestarem uma mudança para os lugares mais privados. Estes aspectos aparecem directamente ligados às transições associadas ao desenvolvimento da amizade heterossexual (no nosso estudo a adesão à actividade «Namorar» aumenta significativamente $\mathrm{p}<0.001)$ verificando-se que essas transições influenciam a mudança consequente nos contextos que os adolescentes seleccionam para as suas actividades de lazer (23).
Mas se o interesse e participação nas relações sexuais emerge, influenciando gradualmente as actividades sociais (16), conforme verificámos, outros resultados não parecem estar totalmente de acordo com o nosso estudo. Isto é, parece que na transição da infância para a adolescência a influência da família deixa de se fazer sentir e o grupo de pares passa a ser mais significativo, particularmente no caso dos pares do mesmo sexo, que passam a funcionar mais como modelos $(2,26,27)$.

Se em relação à diminuição da influência da família podemos observar indirectamente (menor adesão nas actividades "Trabalhos para a escola" - p $<0.001$, maior adesão nas actividades "Namorar» e "Ir à discoteca» - $\mathrm{p}<0.001)$ uma, provável, maior ausência de controlo por parte dos pais em relação aos pares, os nossos resultados não são esclarecedores, dado que a actividade "Conversar com os amigos(as)" não apresenta tendências significativas ao longo da adolescência e «Visitar pessoas conhecidas» apresenta uma tendência significativa $(\mathrm{p}<0.001)$ de não adesão. De qualquer modo, a idade parece ser uma variável importante, associando-se a alterações comportamentais decisivas, e podendo, portanto, influenciar as práticas e escolhas de lazer da população juvenil.

\section{CONCLUSÃO}

Os nossos resultados indicam que os adolescentes têm uma grande componente de tempo livre não estruturado (cerca de $40 \%$ ). Neste contexto parece importante o desenvolvimento de programas que comprometam a sua ocupação de tempos livres com desafios suficientemente estimulantes. As diferenças de género e etárias devem também ser tidas em conta ao nível dos programas de intervenção, no sentido de favorecer práticas tidas como mais positivas no tempo de lazer da população infanto-juvenil.

\section{Agradecimentos}

Este trabalho foi apoiado pela Fundação Calouste Gulbenkian: Proj: 40659/00.

\section{CORRESPONDÊNCIA}

\section{Jorge Mota}

Faculdade de Ciências do Desporto e de Educação

Física, Universidade do Porto

R. Plácido Costa, 91, 4200-450 Porto, Portugal jmota@fcdef.up.pt 


\section{BIBLIOGRAFIA}

1. Albuquerque, T.M.M. (1999) Lazer e desenvolvimento na adolescência numa perspectiva da Psicologia Social: Teoria, investigação e intervenção. Instituto de Educação e Psicologia da Universidade do Minho. [Dissertação de Doutoramento em Psicologia].

2. Brown, B.B.; Eicher, S.A.; Petrie, S. (1986) The importance of peer group (crowd) affiliation in adolescence. Journal of Adolescence, 9: 73-95.

3. Busser, J.A. (1995) Differences in adolescent participation by Gender, Grade and Race. Research Quarterly for Exercise and Sport (Supplement), 66 n.1.

4. Cloes, M.; Ledent, M.; Didier, P.; Diniz, J.; Piéron, M. (1997) Pratique et importance des principales activités de loisirs chez des jeunes de 12 à 15 ans dans cinq pays européens. ADEPS, 159/160:51-60.

5. Cotterell, J.L. (1993) Do macro-level changes in the leisure environment alter leisure constraints on adolescent girls? Journal of Environmental Psychology, 13:125-136.

6. Csikszentmihayi, M.; Larson, R.; Prescott, S. (1977) The ecology of adolescent activity and experiences. Journal of Youth and Adolescence, 6: 281-294.

7. Delle Fave, A. (1996) Esperienza ottimale e sviluppo del comportamento: evidenze sperimrntali. In F. Massimini, P. Inghilleri \& A. Delle Fave (Eds.), La selezione psicologica umana: Teoria e metodo d analisi. Milano: Cooperativa Libraria IULM.

8. Delle Fave, A.; Bassi, M. (1998) Optimal experience and apathy: The meaning of experience fluctuation in adolescents. Comunicação apresentada no 6th Biennial EARA Conference. Budapeste, Hungria.

9. Fine, G.A.; Mortimer, J.T.; Roberts, D.F. (1990) Leisure, Work, and the mass media. In S.S. Feldman \& G.R. Elliot (Eds.) At the Threshold: The developing adolescent. Cambridge, M.A.: Harvard University Press, 225-252.

10. Fitzgerald, M. Joseph, A.P., Hayes, M.; O`Regan, M. (1995) Leisure activities of adolescent schoolchildren. Journal of Adolescence, 18: 349-358.

11. Furlong, A.; Campbell, R.; Roberts, K. (1990) The effects of Post-16 experiences and social class on the leisure patterns of young adults. Leisure Studies, 9: 213-224.

12. Hargraves, J. (1989) The promise and problems of women`s leisure and sport. In C. Rojek (Ed.) Leisure For Leisure: Critical Essays. London: Macmillan, 18-140.

13. Kogan, J. (1974) Creativity and Sex differences. Journal of Creative Behaviour, 8: 1-14.

14. Larson, R. (1994) Youth organisations, hobbies and sports as developmental contexts. In R.K. Silbereisen \& E. Todt (Eds.) Adolescence in context: The interplay of family, school, peers, and work in adjustment. New York: Spinger-Verlag.

15. Martens, R. (1996) Turning Kids on to Physical Activity for a Lifetime. American Academy of Kinesiology and Physical Education, 48: 303-310.

16. Miller, P.Y.; Simon, W. (1980) The development of sexuality in adolescence. In J. Adelson (Ed.) Handbook of adolescent psychology. New York: John Wiley.

17. Pittman, K.J.; Cahill, M. (1991) A new vision: Promoting youth development. Washington, D. C.: Academy for Educational Development.

18. Richards, M.H.; Larson, R. (1989) The life space and socialisation of the self: Sex differences in the young ado- lescent. Journal of youth and Adolescence, 18: 617-626.

19. Roth, J.; Brooks-Gunn, J.; Murray, L.; Foster, W. (1998) Promoting healthy adolescents: Synthesis of youth development program evaluations. Journal of Research on Adolescence, 8 (4): 423-459.

20. Scott, D.; Willits, F.K. (1989) Adolescent and Adult leisure Patterns: A 37- year follow-up study. Leisure Sciences, 11: 323-335.

21. Shaw, S. M., Kleiber, D.; Caldwell, L. (1995) Leisure and identity formation in male and female: A preliminary examination. Journal of Leisure Research, 27: 245-263.

22. Silbereisen, R.K.; Noack, P.; Eyferth, K. (1986) Place for development: Adolescents, leisure settings, and developmental tasks. In R.K. Silbereisen, K. Eyferth \& G. Rudinger (Eds.), Development as action in context. New York: Springer-Verlag.

23. Silbereisen, R.K.; Noack, P.; von Eye, A. (1992) Adolescent's development of romantic friendship and change in favourite leisure contexts. Journal of Adolescence Research, 7: 80-93.

24. Sports Council (1988) Sports in the Community: Into the 90's. A Strategy for Sport 1998-1993. London: Sports Council.

25. Wankel, L.M. (1994) Health and leisure: Inextricably linked. Journal of Physical Education, Recreation \& Dance, 65(4): 28-31.

26. Weiss, M.R.; Ebbeck, V.; Horn, T.S. (1996a) Children's self-perceptions and sources of physical competence information: A cluster analysis. Journal of Sport \& Exercise Psychology, 19: 52-70.

27. Weiss, M.R.; Smith, A.L.; Theeboom, M. (1996b) That's what friends are for: Children's and teenagers ` perceptions of peer relationships in the sport domain. Journal of Sport \& Exercise Psychology, 18: 347-379. 